\title{
A Narrative Review of Alternate Gait Training Using Knee-ankle-foot Orthosis in Stroke Patients with Severe Hemiparesis
}

\author{
Hiroaki Abe, PhD, $\mathrm{PT}^{1}$, Kei Kadowaki, $\mathrm{PT}^{2}$, Naohide TsuJimoto, $\mathrm{PT}^{3}$ and Toru OKAnUKA, $\mathrm{PT}^{4}$ \\ ${ }^{1)}$ Fukushima Medical University School of Health Sciences, Japan \\ ${ }^{2)}$ Department of Rehabilitation, Osaki Citizen General Hospital, Japan \\ ${ }^{3)}$ Department of Rehabilitation Medicine, Nishiyamato Rehabilitation Hospital, Japan \\ ${ }^{4)}$ Department of Rehabilitation Medicine, Kohnan Hospital, Japan
}

\begin{abstract}
Impairments resulting from stroke lead to persistent difficulties with walking. Subsequently, an improved walking ability is one of the highest priorities for people living with stroke. The degree to which gait can be restored after a stroke is related to both the initial impairment in walking ability and the severity of paresis of the lower extremities. However, there are some patients with severe motor paralysis and a markedly disrupted corticospinal tract who regain their gait function. Recently, several case reports have described the recovery of gait function in stroke patients with severe hemiplegia by providing alternate gait training. Multiple studies have demonstrated that gait training can induce "locomotor-like" coordinated muscle activity of paralyzed lower limbs in people with spinal cord injury. In the present review, we discuss the neural mechanisms of gait, and then we review case reports on the restoration of gait function in stroke patients with severe hemiplegia.
\end{abstract}

Key words: Knee-ankle-foot orthosis (KAFO), Stroke, Gait, Hemiparesis, Inverted pendulum

(Phys Ther Res 00: 00-00, 0000)

G lobally, the estimated total number of stroke patients was 33 million in $2010^{1}$. Stroke is the second leading cause of death and a major contributor to disability worldwide ${ }^{2)}$. Impairments resulting from stroke often lead to persistent difficulties with walking. Subsequently, an improved walking ability is one of the highest priorities for people living after a stroke ${ }^{3)}$. In addition, walking ability has important health implications in providing protective effects against secondary complications that are common after a stroke, such as heart disease or osteoporosis ${ }^{3)}$.

The degree to which gait can be restored after a stroke is related to both the initial impairment in walking ability and the severity of paresis of the lower extremities ${ }^{4-8}$. Wan-

Received: August 25, 2021

Accepted: September 15, 2021

Advance Publication by J-STAGE: December 6, 2021

Correspondence to: Hiroaki Abe, Fukushima Medical University School of Health Sciences, Japan, 10-6 Sakaemachi, Fukushima city, 960-8516, Japan

\# e-mail: abe-hrk@fmu.ac.jp

doi: 10.1298/ptr.R0015 del et al. ${ }^{7)}$ reported that only $21 \%$ of stroke survivors with lower limb paralysis regained the ability to walk. In other words, there is no doubt that severe paralysis of the lower limbs and muscle weakness are factors closely related to the ability to walk. However, there are patients with severe motor paralysis and a markedly disrupted corticospinal tract who regained their gait function ${ }^{9,10)}$. In addition, Sivaramakrishnan and Madhavan ${ }^{11)}$ reported that there was no association between transcranial magnetic stimulation-induced tibialis anterior and rectus femoris motor evoked potentials, which are neurophysiological parameters of lower limb function, and walking speeds. Indeed, many patients have regained gait function even after experiencing severe hemiparesis $^{12-16)}$. Recently, several case reports ${ }^{12-14)}$ described the restoration of gait function in stroke patients with severe hemiplegia after the provision of alternate gait training (AGT). Multiple studies have demonstrated that gait training can induce "locomotor-like" coordinated muscle activity of the paralyzed lower limbs in people with spinal cord injury $^{17-19)}$.

In the present narrative review, we will discuss the 
neural mechanisms of gait, and then review case reports on the restoration of gait function in stroke patients with severe hemiplegia.

\section{Alternative Gait Training Using Knee-ankle-foot Orthosis (KAFO) for Stroke Patients with Severe Hemiplegia}

1) Neural mechanisms of gait, and ideas for restoring gait in stroke patients with severe hemiplegia.

Decerebrate cats, with an absence of the forebrain, can walk, trot and gallop. When decerebration occurs at precollicular-postmammillary level, the cat initiates locomotion by electrical or chemical stimulation applied to the mesencephalic or midbrain locomotor region ${ }^{20-23)}$. To date, three locomotor regions have been identified in animals: the midbrain locomotor region in the mesopontine tegmentum, the subthalamic locomotor region, and the cerebellar locomotor region in the mid-part of the cerebellum ${ }^{24}$. Human imaging has demonstrated that the organization of these supraspinal locomotor centers was preserved during the transition to bipedal locomotion in humans ${ }^{25}$.

The regulation of human upright posture and locomotion is based on the finely tuned coordination of muscle activation between the two legs ${ }^{17)}$. For example, when a disturbance causes an initiation or prolongation of the swing phase on one side, the stance phase of the contralateral leg compensates accordingly, in both human infants and cats ${ }^{17}$. Unilateral leg displacement during stance and gait evoke a bilateral response pattern with similar short (i.e., spinal) onset latencies on both sides ${ }^{17)}$. This interlimb coordination is necessary to keep the body's center of gravity over the feet $^{17,26)}$

In cats, there are two main sources of afferent input that lead to rhythm entrainment and/or resetting of locomotor activity ${ }^{17)}$. Such input can either block or induce switching between the alternating flexor and extensor locomotor bursts. One afferent input source is related to hip position, and the other is related to load ${ }^{17,27)}$. For example, for the initiation of the swing phase, the significance of hip position was essential for human infant stepping, similarly to what was described for the cat with chronic spinal cord transec$\operatorname{tion}^{17)}$. Furthermore, in previous studies, load receptor input for the regulation of stance and gait was important for cats ${ }^{28)}$ and humans ${ }^{29)}$. It was assumed that this effect was mediated by group Ib afferent input ${ }^{30)}$.

Further studies have indicated that even in completely paraplegic patients, a locomotor pattern can be evoked by bilateral alternating stepping ${ }^{31,32)}$. In these studies, load receptor input was essential for leg muscle activation during stepping movements ${ }^{17)}$. Habli and Dietz ${ }^{19)}$ found that loadand hip-joint- related afferent input is of crucial importance during locomotor training, as it leads to appropriate leg muscle activation, and thus increases the efficacy of reha- bilitative training. According to previous reports, gait function is influenced by the following: the control system of voluntary function, such as the corticospinal tract; the control system of involuntary motor function, such as the mesencephalic-reticulospinal neuron; and the central pattern generator in the spinal cord ${ }^{33-35)}$.

In patients with spinal cord injury, alternate stepping movements with afferent input from load receptors induce a patterned leg muscle activation similar to that induced in healthy subjects ${ }^{17,18)}$. In other words, these inputs induce lower limb muscle activity in patients with difficulties in voluntary lower limb movement. Therefore, we hypothesized that earlier improvement of gait function could be achieved by providing an alternate gait pattern for patients with severe hemiplegia, because it facilitates the afferent load and proprioceptive receptor inputs. In particular, afferent information from both bilateral hip joints seems to be essential for the generation of locomotor-patterned leg muscle activation; however, unilateral stepping movements lead to inadequate leg muscle activation ${ }^{17,18)}$. Indeed, patients with severe hemiparesis commonly have poor lower limb stability on the paretic side and difficulty in walking without lower limb support. To achieve good stability, patients benefit from strong external support, such as a KAFO, which provides both stability and enables the ankle to perform alternate stepping. We hypothesized that patients may regain gait function earlier if gait training is implemented using a KAFO. We defined AGT as walking with alternate large hip flexion and extension when using a KAFO with an oil damper ankle hinge.

\section{2) Technique for providing AGT using a KAFO with an oil damper ankle hinge}

A KAFO for use in AGT has a ring lock hinge for the knee joint and an oil damper hinge for the ankle joint ${ }^{36,37)}$. (Fig. 1) The hinged oil damper can resist plantarflexion during swing, and produce plantarflexion after heel con$\operatorname{tact}^{36-38)}$. Therefore, the loading response period is properly constructed because the first rocker function is maintained $^{36-38)}$. Moreover, using a KAFO with an oil damper ankle hinge has other merits as it matches the inverted pendulum movement that is observed in normal walking (Fig. 2A, B) because the hinged oil damper does not hinder dorsiflexion $^{36-38)}$. An inverted pendulum model of the stance leg is crucial for effective, economical, and stable ambulation in biomechanical features ${ }^{39)}$. AGT is provided by a physical therapist (Fig. 2C), and if AGT cannot be performed with therapists providing external assistance, step training is utilized for each of the affected and unaffected lower limbs. AGT is then started shortly after step training. After resolution of the problem of knee instability, an ankle-foot orthosis (AFO) is used. Before transitioning from KAFO to AFO (known as "cutting down"), we confirm that subjects can walk with an alternate gait pattern with AFO alone. 

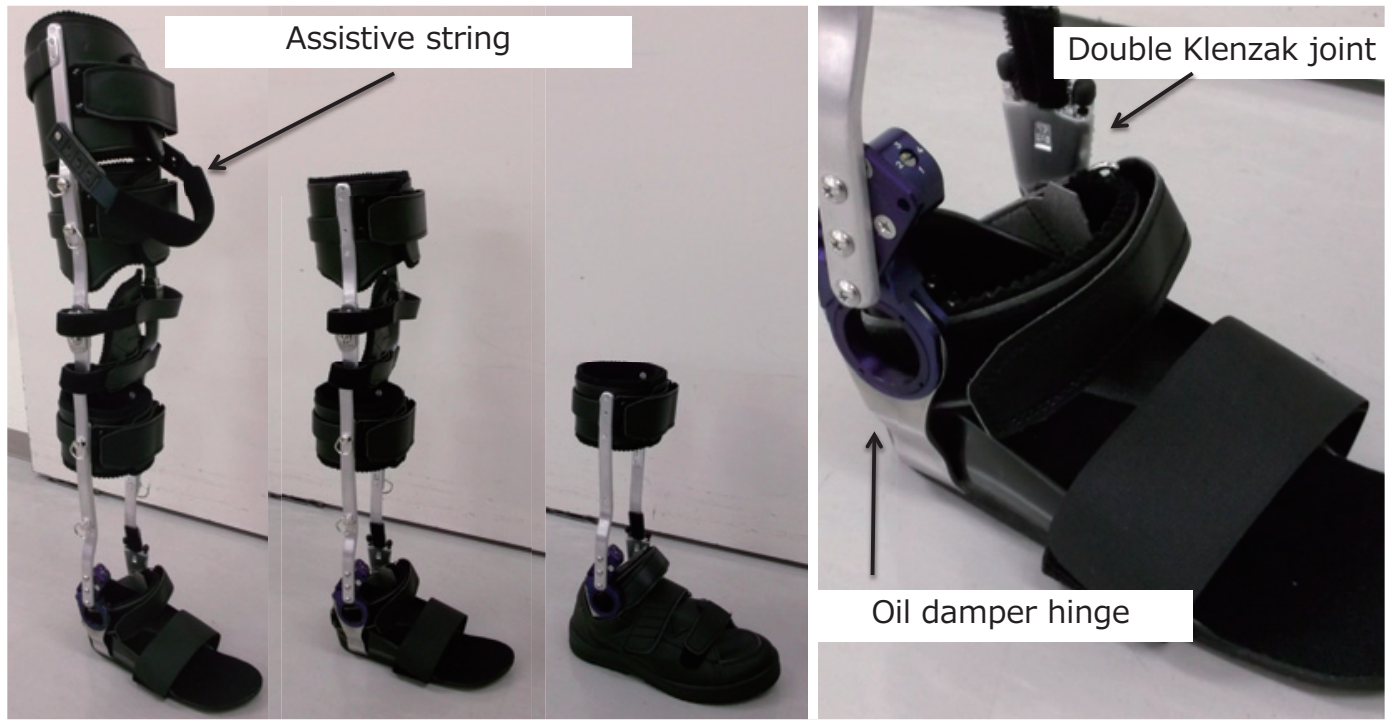

Fig. 1. A knee-ankle-foot orthosis with an oil damper ankle hinge.

The hinged oil damper can resist plantarflexion during swing and promote adequate plantarflexion after heel contact.
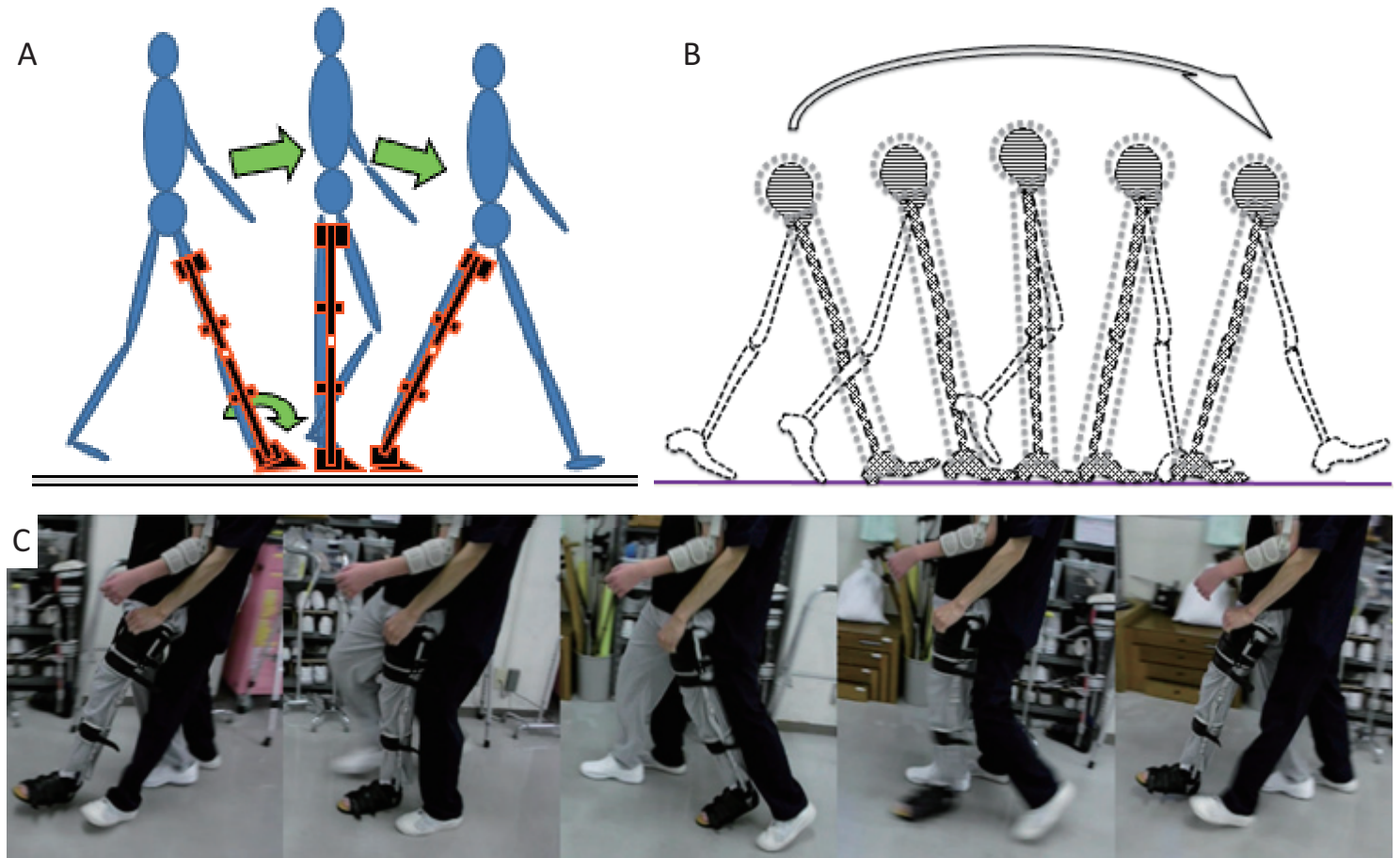

Fig. 2. Alternate gait pattern using knee-ankle-foot orthosis with an oil damper ankle hinge that matches the inverted pendulum model.

A: Alternate gait pattern using knee-ankle-foot orthosis with an oil damper ankle hinge

B: The inverted pendulum model

C: Alternate gait pattern using knee-ankle-foot orthosis with an oil damper ankle hinge with external assist by physiotherapist 


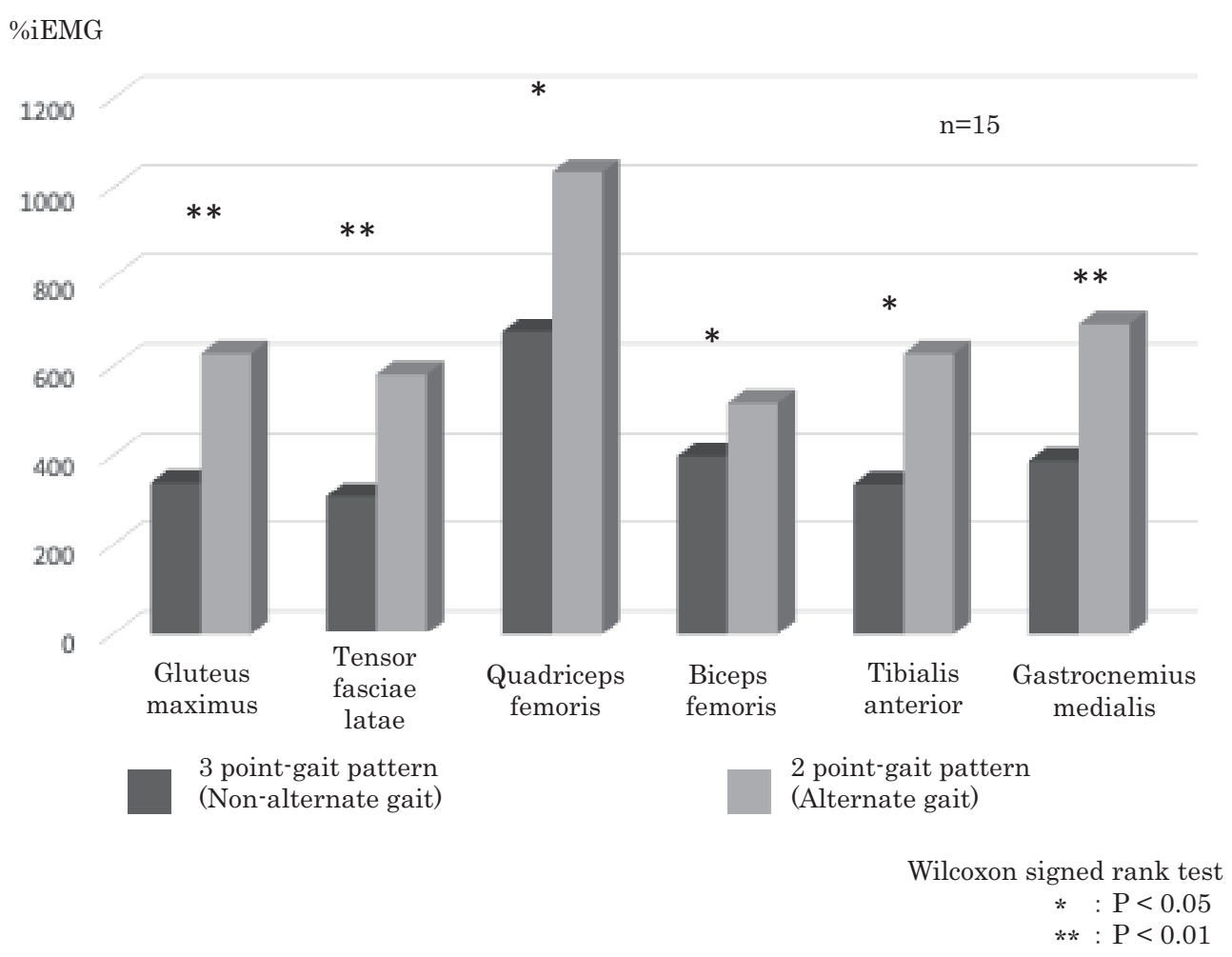

Fig. 3. Electromyography results

Integrated electromyography revealed that all six muscles had more increased muscle activity in the affected side lower limb during the stance phase of the 2-point gait pattern than of the 3-point gait pattern.

\section{A Comparative Study of the Effect of AGT Using a KAFO}

1) Muscle activity during AGT; the differences in lower leg muscle activity between 2-point gait (AGT pattern) and the conventional 3-point gait patterns (defined as walking with a stride length of the non-affected leg not exceeding that of the affected leg).

We have investigated ${ }^{40)}$ differences in lower limb muscle activity during two gait patterns - 2-point gait without a cane and 3-point gait with a cane-in 12 stroke patients. All 12 patients required KAFOs due to severe hemiparesis when walking. When performing the 2-point gait training, the patients would step the unaffected foot in front of the affected foot with assistance provided by a physical therapist. In contrast, when carrying out the 3-point gait training, patients would step the unaffected foot to the lateral side of the affected foot using the cane with slight assistance by the physical therapist. The muscle activities were measured by electromyogram in the tibialis anterior, gastrocnemius medialis, biceps femoris, quadriceps femoris, gluteus maximus, and tensor fasciae latae muscles of the affected lower limb. Integrated electromyography revealed that all six muscles had increased muscle activity during the stance phase of the 2-point gait pattern than of the 3-point gait pattern (Fig. 3). In severe hemiparetic patients, 2-point gait training may be more effective in facilitating muscle activity of the paretic lower limb than 3-point gait training.

\section{2) AGT with a KAFO to support earlier walking independ- ence}

We have previously reported ${ }^{41)}$ the effects of physical therapy with an early prescription of KAFO during the course of recovery of walking and stair climbing functions evaluated using Functional Independence Measures (FIM). Eight patients with post-stroke hemiparesis, who had been prescribed a KAFO and had received AGT during their acute hospital stays (the KAFO group), and 20 patients who had not been prescribed a KAFO (the non-KAFO group), were recruited. All patients in the non-KAFO group had similar characteristics to those in the KAFO group on admission; age, length of hospitalization, severity of hemiparesis, duration between stroke onset and admission to the convalescent rehabilitation ward, walking function, and stair climbing function. We compared the time course of recovery of walking and stair climbing functions between the KAFO and non-KAFO groups by using FIM at admission, midpoint of the hospital stay, and discharge from the rehabilitation ward. FIM for walking and stair climbing gradually improved in both groups. However, walking function improved earlier in the KAFO group than in the non-KAFO group (Fig. 4A). Furthermore, the stair climbing function at 
A

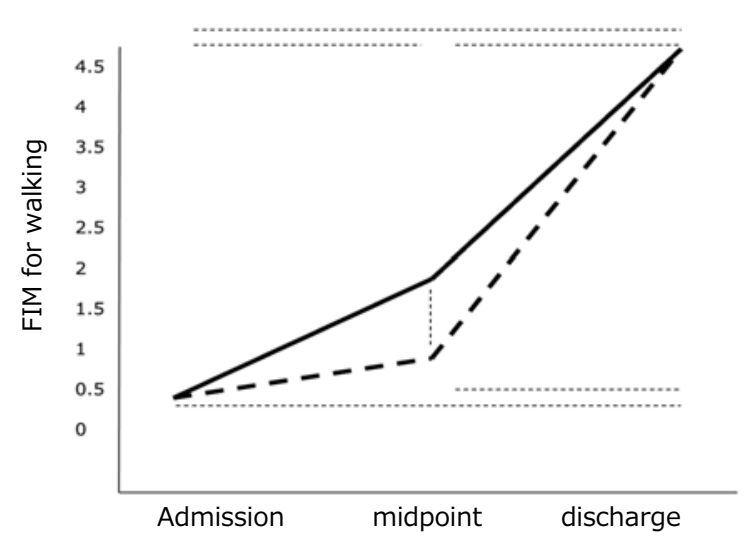

Bulletin of the Japanese Society of Prosthetics and Orthotics. 34(1), 52-59.

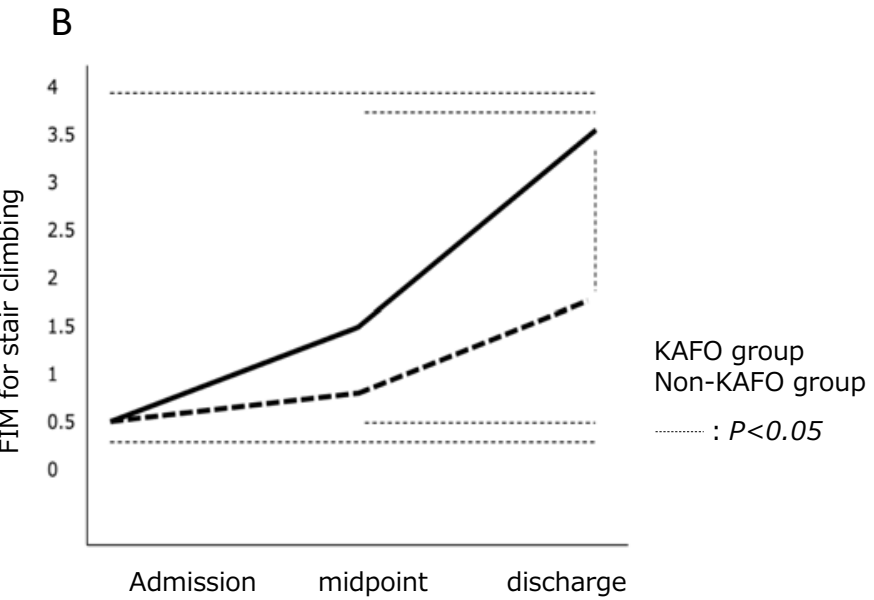

Admission midpoint discharge
KAFO group

Non-KAFO group

Fig. 4. The time course of recovery of walking and stair climbing functions in the KAFO and non-KAFO groups evaluated using FIM at admission, midpoint of hospital stay, and discharge.

discharge was significantly better in the KAFO group than in the non-KAFO group (Fig. 4B). Therefore, physical therapy using an early-prescribed KAFO might be effective for improving walking and stair climbing functions in poststroke patients with severe hemiparesis.

\section{Case Reports}

1) Gait restoration in a patient who required full assistance to walk at 6 months after stroke onset

Kadowaki et al. ${ }^{12)}$ tried to restore the gait function in a patient with severe stroke hemiparesis who required full assistance to walk 6 months after stroke onset. This patient was a woman in her $50 \mathrm{~s}$ who had suffered subarachnoid and intracerebral hemorrhages. Her Brunnstrom motor function was stage II in the upper limbs and fingers, and IIIII in the lower limbs.

The patient underwent physical therapy in the Kaifukuki rehabilitation (convalescent) ward and received gait training using a soft knee brace and a soft bandage between 75 and 176 days of stroke onset. At six months post-stroke, any improvement in motor paralysis was unlikely. Nevertheless, we assumed that her gait function could be improved if lower limb muscle activity was induced by providing AGT.

Initially, we provided AGT using a soft knee orthosis and an AFO. However, the inverted pendulum movement could not be constructed because of the bent knee of the affected side during the stance phase. We therefore decided to make a KAFO for the patient. Figure $5 \mathrm{~A}$ shows the gait pattern at 3 weeks after admission. Figure 5B shows a trial transition from the KAFO to the AFO. In this trial, the knee joint was observed as too bent in the AFO, we this continued gait training using the KAFO. Figure 5C shows the patient's gait at discharge, and Figure 5D shows the gait at home following hospital discharge. The patient achieved re- markable recovery with AGT; from being unable to walk at 6 months after stroke onset to eventually being able to walk independently.

2) Gait reconstruction in a patient with complete damage to the corticospinal tract.

We (Tsujimoto et al. ${ }^{13)}$ ) previously reported a case of gait reconstruction in a young patient with large right frontal lobe cortical hemorrhage due to a ruptured arteriovenous malformation (AVM). The lesion extended from the central precentral gyrus to the subcortical region of the postcentral gyrus (Fig. 6A). It was not possible to visualize the corticospinal and sensory tracts on diffusion tensor tractography (Fig. 6B). The patient in fact showed severe hemiparesis and severe sensory disturbance. The patient's functional ambulation category (FAC) went from 5 (fully independent) before the AVM ruptured to FAC 0 (unable to walk).

The patient's knee was excessively bent and showed an extension thrust pattern (Fig. 7A). For restoration of his gait function, a KAFO with the oil damper ankle hinge was used, and AGT was provided. Initially, the patient was assisted by a physiotherapist (Fig. 7B), with the assistance gradually reduced (Fig. 7C). A previous report ${ }^{9}$ stated that gait reconstruction is possible even when the corticospinal tract is completely damaged. In this young patient, physical therapy was started on Day 34 after the AVM rupture, and his walking ability gradually improved, reaching $58.8 \mathrm{~m} /$ min as a maximum walking speed on Day 113 (Fig. 7D). At the same time, the KAFO was switched over to an AFO, and his gait training continued. After transfer to a convalescent hospital, the patient was eventually able to walk independently both indoors and outdoors.

\section{Conclusions}

In the current narrative review, we introduced AGT 

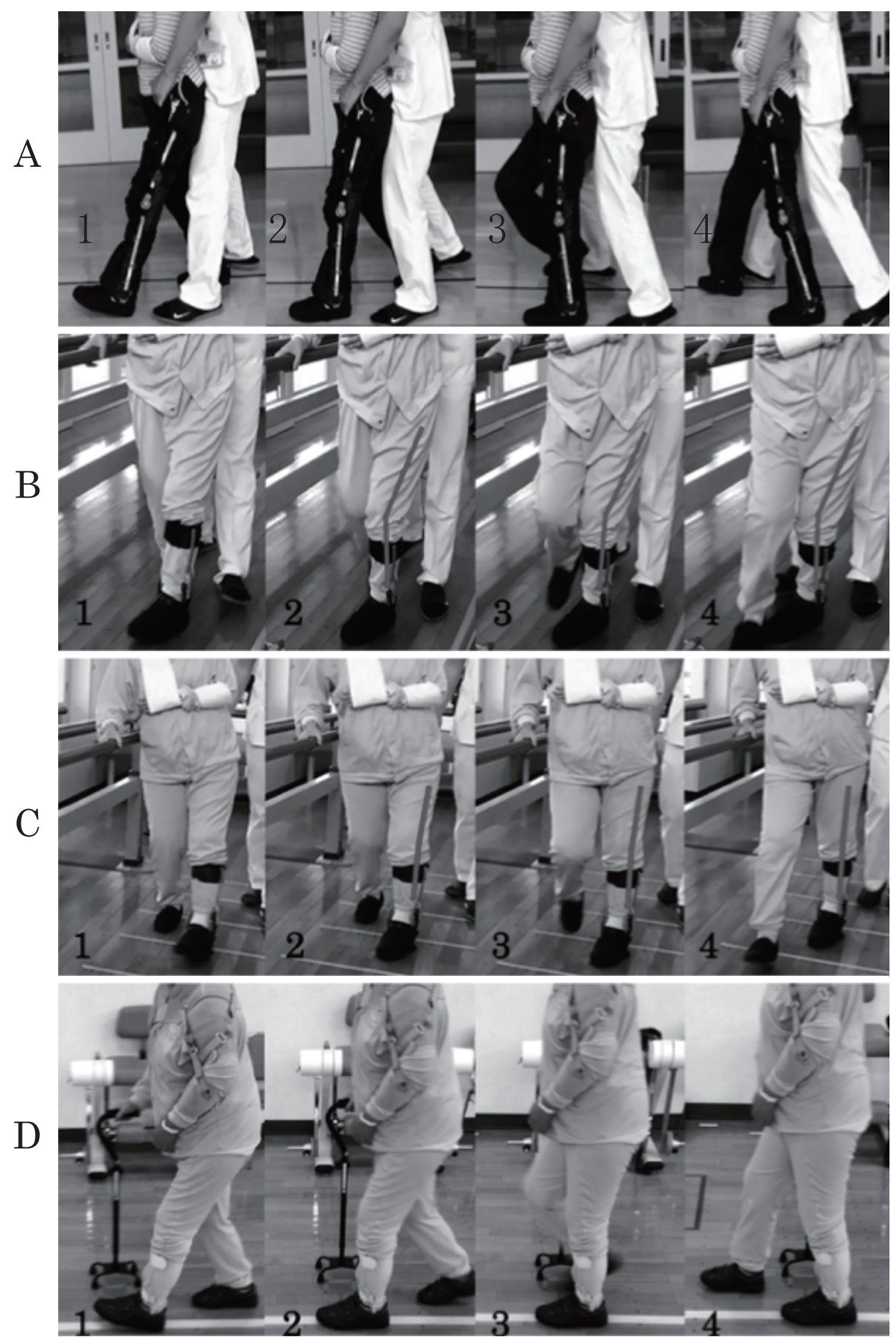

Fig. 5. The restoration of gait function in a stroke patient with severe hemiparesis who required external assistance for gait 6 months after stroke onset.

1. Initial contact; 2. Loading response; 3 . Mid stance; 4. Terminal stance 


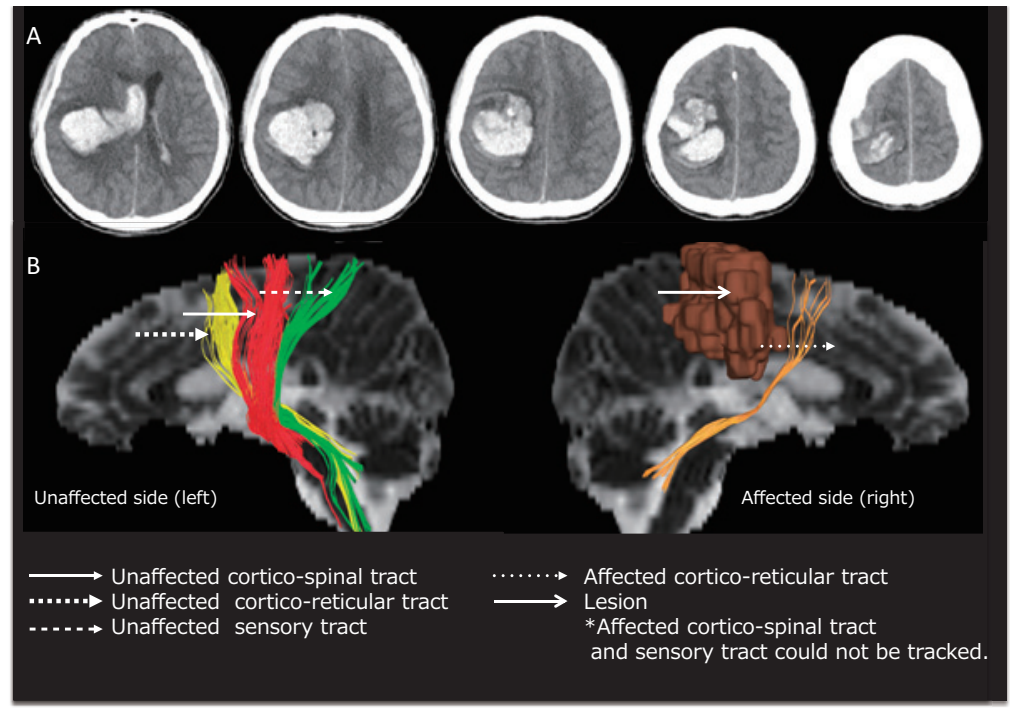

Fig. 6. Brain imaging of intracranial hemorrhage due to ruptured arteriovenous malformation.

A: Computed tomography

B: Diffusion tensor tractography

A

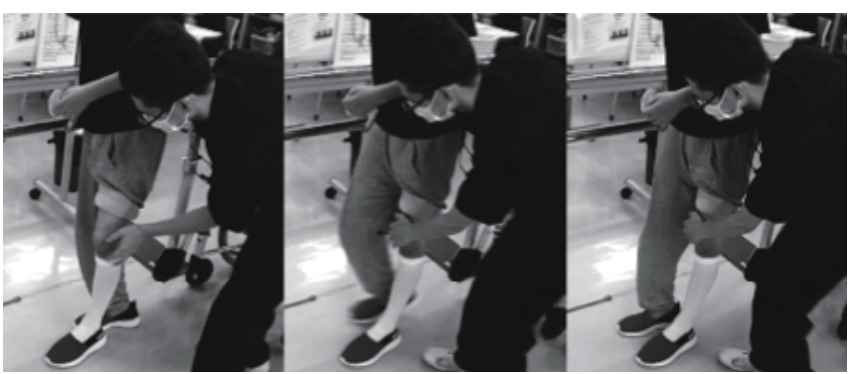

$\mathrm{B}$

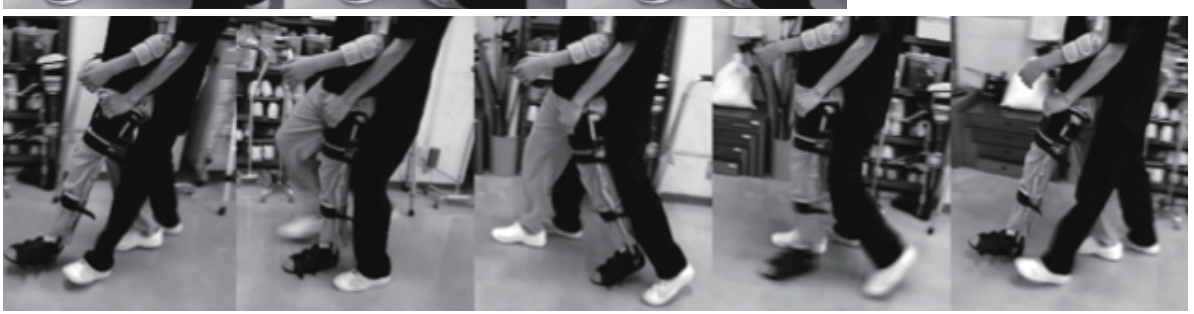

C

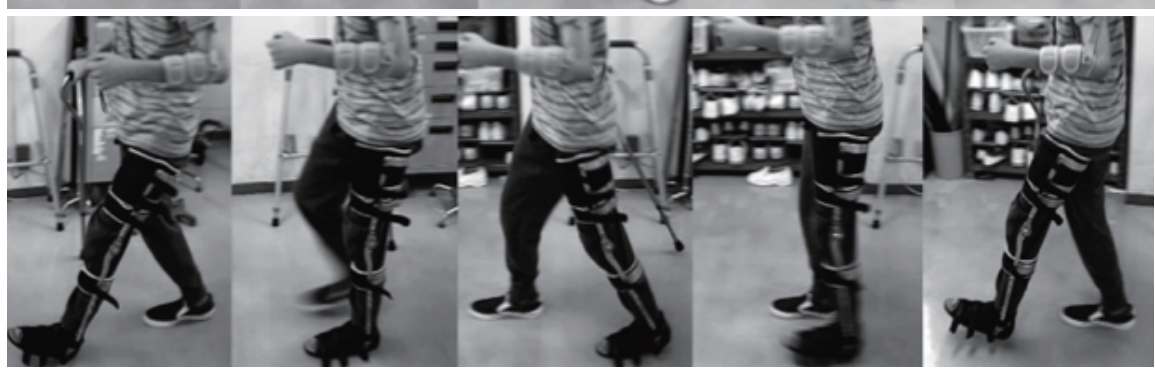

$\mathrm{D}$

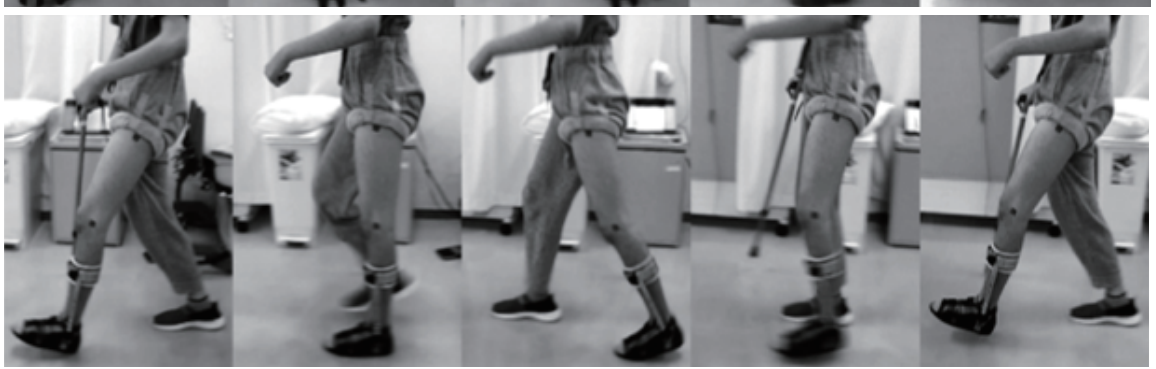

Fig. 7. The restoration of gait function in a stroke patient with severe hemiparesis with complete disconnection of the corticospinal tract and sensory tract on the affected side. 
using a KAFO for restoration of gait function in stroke patients with severe hemiparesis. We believe that AGT with a KAFO can benefit stroke patients with severe hemiparesis as they may achieve earlier improvements in gait ability. Neurological physical therapists should be encouraged to use this approach for restoration of gait function in such patients. However, the effect of AGT is not fully elucidated, and there are very few reports on this subject, all of which are written in Japanese. Further study is required to validate the effect of AGT with a KAFO.

Conflict of Interest: The authors declare that there are no conflicts of interest.

\section{References}

1) $\mathrm{Ng} \mathrm{M}$, Fleming T, et al.: Global, regional, and national prevalence of over-weight and obesity in children and adults during 1980-2013: a systematic analysis for the Global Burden of Disease Study 2013. Lancet. 2014; 384: 766-781.

2) Kuriakose D and Xiao Z: Pathophysiology and Treatment of Stroke: Present Status and Future Perspectives. Int J Mol Sci. 2020; 21: 7609.

3) Eng JJ and Tang PF: Gait training strategies to optimize walking ability in people with stroke: a synthesis of the evidence. Expert Rev Neurother. 2007; 7: 1417-1436.

4) Mercer VS, Freburger JK, et al.: Recovery of paretic lower extremity loading ability and physical function in the first six months after stroke. Arch Phys Med Rehabil. 2014; 95: 15471555.

5) Hirano Y, Hayashi T, et al.: Prediction of Independent Walking Ability for Severely Hemiplegic Stroke Patients at Discharge from a Rehabilitation Hospital. J Stroke Cerebrovasc Dis. 2016; 25: 1878-1881.

6) Taylor-Piliae RE, Latt LD, et al.: Predictors of gait velocity among community-dwelling stroke survivors. Gait Posture. 2012; 35: 395-399.

7) Wandel A, Jørgensen HS, et al.: Prediction of walking function in stroke patients with initial lower extremity paralysis: the Copenhagen Stroke Study. Arch Phys Med Rehabil. 2000; 81: 736738.

8) Kluding P and Gajewski B: Lower-extremity strength differences predict activity limitations in people with chronic stroke. Phys Ther. 2009; 89: 73-81.

9) Ahn YH, Ahn SH, et al.: Can stroke patients walk after complete lateral corticospinal tract injury of the affected hemisphere? Neuroreport. 2006; 17: 987-990.

10) Cho HM, Choi BY, et al.: The clinical characteristics of motor function in chronic hemiparetic stroke patients with complete corticospinal tract injury. NeuroRehabilitation. 2012; 31: 207213.

11) Sivaramakrishnan A and Madhavan S: Absence of a Transcranial Magnetic Stimulation-Induced Lower Limb Corticomotor Response Does Not Affect Walking Speed in Chronic Stroke Survivors. Stroke. 2018; 49: 2004-2007.
12) Kadowaki K, Abe H, et al.: A case with severe hemiplegia who regained gait function due to proceeded gait exercise using a Knee-Ankel-Foot Orthosis since the state requiring assistance for gait at 6 moths after the stroke onset. (in Japanese) Physical Therapy Japan. 2018; 45: 183-189.

13) Tsujimoto $\mathrm{N}$, Abe $\mathrm{H}$, et al.: The practice of aggressive gait training using Knee-Ankel-Foot Orthosis ad the course of recovery from gait disturbance and weakness of proximal lower muscles in severe hemiplegic stroke patient with intact cortico-reticular tracts. (in Japanese) Physical Therapy Japan. 2019; 46: 285-392.

14) Kadowaki $\mathrm{K}$, Abe $\mathrm{H}$, et al.: Improvement of walking ability by practicing gait training using an orthosis intended to reconstruct an inverted pendulum model in two hemiplegic patients. (in Japanese) Physical Therapy Japan. 2019; 46: 38-46.

15) Abe H, Okanuka T, et al.: Gait Training for Severe Hemiplegia in Acute Stroke. (in Japanese) Annual report of the Miyagi Physical Therapy Association. 2016; 27: 17-27.

16) Abe H, Tsujimoto N, et al.: Gait Training for Severe Hemiplegia in Acute Stroke 2nd. (in Japanese) Annual report of the Miyagi Physical Therapy Association. 2017; 28: 11-20.

17) Dietz V, Müller R, et al.: Locomotor activity in spinal man: significance of afferent input from joint and load receptors. Brain. 2002; 125(Pt 12): 2626-2634.

18) Kawashima N, Nozaki D, et al.: Alternate leg movement amplifies locomotor-like muscle activity in spinal cord injured persons. J Neurophysiol. 2005 Feb; 93: 777-785.

19) Hubli $M$ and Dietz $V$ : The physiological basis of neurorehabilitation--locomotor training after spinal cord injury. $\mathrm{J}$ Neuroeng Rehabil. 2013 Jan 21; 10: 5.

20) Takakusaki K: Functional Neuroanatomy for Posture and Gait Control. J Mov Disord. 2017 Jan; 10: 1-17.

21) Takakusaki K: Forebrain control of locomotor behaviors. Brain Res Rev. 2008; 57: 192-198.

22) Mori S: Integration of posture and locomotion in acute decerebrate cats and in awake, freely moving cats. Prog Neurobiol. 1987; 28: 161-195.

23) Armstrong DM: Supraspinal contributions to the initiation and control of locomotion in the cat. Prog Neurobiol. 1986; 26: 273361.

24) Mori S, Matsui T, et al:: Stimulation of a restricted region in the midline cerebellar white matter evokes coordinated quadrupedal locomotion in the de-cerebrate cat. J Neurophysiol. 1999; 82: 290-300.

25) Jahn K, Deutschländer A, et al.: Imaging human supraspinal locomotor centers in brainstem and cerebellum. Neuroimage. 2008; 39: 786-792.

26) Dietz V: Human neuronal control of automatic functional movements. Interaction between central programs and afferent input. [Review]. Physiol Rev. 1992; 72: 33-69.

27) Dietz V and Duysens J: Significance of load receptor input during locomotion. [Review]. Gait Posture. 2000; 11: 102-110.

28) Prochazka A, Gillard D, et al.: Positive force feedback control of muscles. J Neurophysiol. 1997; 77: 3226-3236.

29) Dietz V, Gollhofer A, et al.: Regulation of bipedal stance: dependency on 'load' receptors. Exp Brain Res. 1992; 89: 229- 
231.

30) Dietz V: Evidence for a load receptor contribution to the control of posture and locomotion. Neurosci Biobehav Rev. 1998 Jul; 22(4): 495-499.

31) Dietz V, Colombo G, et al.: Locomotor capacity of spinal cord in paraplegic patients. Ann Neurol. 1995; 37: 574-582.

32) Harkema SJ, Hurley SL, et al:: Human lumbosacral spinal cord interprets loading during stepping. J Neurophysiol. 1997; 77: 797-811.

33) Takakusaki K, Kohyama J, et al.: Medullary reticulospinal tract mediating the generalized motor inhibition in cats: parallel inhibitory mechanisms acting on motoneurons and on interneuronal transmission in reflex pathways. Neuroscience. 2001; 103: 511-527.

34) Ballermann M and Fouad K: Spontaneous locomotor recovery in spinal cord injured rats is accompanied by anatomical plasticity of reticulospinal fibers. Eur J Neurosci. 2006; 23: 1988-1996.

35) Matsuyama K, Mori F, et al. : Locomotor role of the corticoreticular-reticulospinal-spinal interneuronal system. Prog Brain Res. 2004; 143: 239-249.

36) Yamamoto $S$, Ibayashi $S$, et al.: Immediate-term effects of use of an ankle-foot orthosis with an oil damper on the gait of stroke patients when walking without the device. Prosthet Orthot Int.
2015; 39: 140-149.

37) Yamamoto S, Tomokiyo N, et al.: Effects of plantar flexion resistive moment generated by an ankle-foot orthosis with an oil damper on the gait of stroke patients: a pilot study. Prosthet Orthot Int. 2013; 37: 212-221.

38) Ohata K, Yasui T, et al.: Effects of an ankle-foot orthosis with oil damper on muscle activity in adults after stroke. Gait Posture. 2011; 33: 102-107.

39) Kuo AD and Donelan JM: Dynamic principles of gait and their clinical implications. Phys Ther. 2010 Feb; 90: 157-174.

40) Okanuka T, Abe H, et al.: Differences in lower limb muscle activity during different gait training pattern between 2-point gait training using KAFO whose unlimited dorsiflexion without a cane and 3-point gait training using KAFO whose limited dorsiflexion with a cane in patients with severe hemiparesis. (in Japanese) Annual Report of The Tohoku Section of Japanese Physical Therapy Association. 29: 20-27.

41) Takashima $Y$ and Abe $H$ : The effect of early construction of a knee-ankle-foot-orthosis during the acute phase in severe hemiplegic patients for functional in-dependence measure of walking and stair climbing. (in Japanese with English abstract) Bulletin of the Japanese Society of Prosthetics and Orthotics. 34(1): 5259. 